\title{
Clinicians' overestimation of febrile child risk assessment
}

\author{
Evelien deVos-Kerkhof ${ }^{1} \cdot$ Damian Roland $^{2} \cdot$ Esther de Bekker-Grob $^{3}$ • \\ Rianne Oostenbrink $^{1}$ - Monica Lakhanpaul ${ }^{4}$ - Henriëtte A. Moll ${ }^{1}$
}

Received: 19 September 2015 / Accepted: 6 November 2015 / Published online: 4 December 2015

(C) The Author(s) 2015. This article is published with open access at Springerlink.com

\begin{abstract}
We aimed to estimate clinicians' based risk thresholds at which febrile children would be managed as serious bacterial infections (SBI) to determine influencing characteristics and to compare thresholds with prediction model (Feverkidstool) risk estimates. Twenty-one video vignettes of febrile children visiting the emergency department (ED) were assessed by 42 (40.4\%) international paediatricians/paediatric emergency clinicians. Questions were related to clinical risk scores of the child having SBI and SBI management decisions on visual analogue scales.
\end{abstract}

Communicated by Peter de Winter

Henriëtte A. Moll

h.a.moll@erasmusmc.nl

Evelien deVos-Kerkhof

e.kerkhof@erasmusmc.nl

Damian Roland

dr98@leicester.ac.uk

Esther de Bekker-Grob

e.debekker@erasmusmc.nl

Rianne Oostenbrink

r.oostenbrink@erasmusmc.nl

Monica Lakhanpaul

m.lakhanpaul@ucl.ac.uk

1 Department of General Paediatrics, Erasmus MC-Sophia Children's Hospital, Wytemaweg 80 room Sp-1541, 3015

CN Rotterdam, The Netherlands

2 Department of Health Sciences, SAPPHIRE Group, Leicester University and Leicester Hospitals, Leicester, UK

3 Department of Public Health, Centre for Medical Decision Making, Erasmus University Medical Centre Rotterdam, Rotterdam, The Netherlands

4 Department of General and Adolescent Paediatrics, UCL Institute of Child Health, Great Ormond Street, London, UK
Feverkidstool risk scores were based on clinical signs/symptoms and C-reactive protein. Amongst vignettes assigned to SBI management, the median risk was $60 \%$ (interquartile range (IQR) $30.0-80.5$ ) and $16.0 \%$ (IQR 5.0-32.0) when vignettes were not managed as SBI. Ill appearance and aberrant circulatory signs were the most influencing factors, as age and duration of fever were the least influencing factors on SBI management decisions. Feverkidstool risk scores varied from $13 \%$ (IQR 7.7-28.1) for SBI management to $7.3 \%$ (IQR 5.7-16.3) for no SBI management.

Conclusion: Clinicians assigned high risk scores to children who they would have managed as SBI, mostly influenced by ill appearance and aberrant circulation. In contrast to SBI risk assessment of the Feverkidstool, clinicians' appeared to apply a more stepwise assessment of the risk of presence/absence of SBI at different steps in the diagnostic and therapeutic process. Uniform risk thresholds at which one should start SBI management in febrile children remains unclear; risk thresholds at which we refrained from SBI management were more consistent.

\section{What is Known:}

- Only a small proportion of febrile children presenting to the emergency department will have serious bacterial infections (SBI) and uniform risk thresholds to start or withhold SBI treatment are not known.

- The low prevalence of SBI and consequently the low exposure of clinicians to these infections make them rely more on alarming signs or clinical decision rules.

\section{What is New:}

- Previously identified model predictors for SBI appeared to be significantly influencing factors in clinicians' febrile child management in emergency care.

- Clinicians' wielded higher risk thresholds regarding SBI febrile child management than reflected by the clinical prediction model. 
Keywords Children · Clinical prediction model · Emergency department $\cdot$ Fever $\cdot$ Serious bacterial infections

\author{
Abbreviations \\ CI confidence interval \\ CRP C-reactive protein \\ ED emergency department \\ SBI serious bacterial infections \\ VAS visual analogue scale
}

\section{Introduction}

The febrile child is a common presentation to emergency departments (ED) with 10 to $20 \%$ of all paediatric patients due to febrile illness alone $[14,17,28]$. Most children suffering from simple self-limiting infections do not need treatment. However, a small proportion will have serious bacterial infections (SBI) which require investigation, hospital admission, antibiotics and in some cases intensive care admission.

Understanding health care professionals' decision making, particularly regarding to diagnosis, treatment and follow-up is of vital importance, particularly as ED's become increasingly overcrowded [33, 34]. Moreover, diagnostic errors, especially in infectious diseases, are amongst the most common medical misadventures of malpractice lawsuits in paediatrics [16].

To support decision making in febrile children, different clinical prediction models have been developed in the past decade $[4,7,12,19,30,31]$. Although most studies on prediction models report good accuracy and high compliance, implementation in paediatric emergency care is limited. One of the reasons might be that clinicians' intuitive estimation of probabilities may be as good as, or better than, prediction models [15, 21, 27]. Moreover, the lack of evidence on clinically based decision thresholds makes the application process of prediction models in clinical practice complex.

The aim of this study was to estimate risk thresholds at which children would be managed as SBI according to clinicians' judgement by assessment of video vignettes of febrile children visiting the ED. Secondary measures included determining the effect of investigations by recording risk estimations after information on C-reactive protein value, determining the presenting characteristics that influence these risks and comparing clinician perceived risk with risk estimates using a validated prediction model (Feverkidstool) [19].

\section{Methods}

\section{Study design and setting}

We performed a cross-sectional study with real life video vignettes of febrile children who presented themselves to the children's ED of the Leicester Royal Infirmary in Leicester, UK. All parents had given formal consent for the video images to be viewed by healthcare professionals under trust policy guidelines via previously published process [13]. Ethical consent for the collection of video images process had been granted by the National Research Ethics Committee East Midlands.

\section{Study population}

Paediatricians and paediatric emergency clinicians from the source population of the REPEM network (Research in Paediatric Emergency Medicine, Europe; www.pemdatabase.org/ REPEM.html), and Paediatricians at teaching hospitals with an interest in acute and emergency care in the Netherlands and United Kingdom, were invited (104 invitations). Nonresponders were sent reminders at 4-week intervals, for a maximum of four mailings per subject.

\section{Study intervention—video vignettes}

Twenty-one online video vignettes of febrile children were shown to the study participants. The vignettes were a mix of children in different age categories with potential SBI and children with simple self-limiting problems reflecting the different levels of severity in febrile child presentations in practice. The videos, with a mean duration of about $30 \mathrm{~s}$, were originally recorded for educational purposes of paediatricians in training as part of the REMIT (Refining Evaluation Methodologies for Practice Changing Interventions) study (ISRCTN94772165). Background history and vital signs were reported as added text or could easily be interpreted from the video vignettes.

Initially, the participants were asked if they should manage the febrile child as having a SBI based on the vignette and background history (e.g. duration of fever) alone. Next, they were asked to assess the actual risk of the child having a SBI on a visual analogue scale $\left(\mathrm{VAS}^{1}\right)$. Finally, we add different values of C-reactive protein (CRP) and asked if their risk assessment would have changed $\left(\mathrm{VAS}^{2}\right)$. The online vignettes and the respondents were hosted on a secure password protected server.

\section{Data collection}

All data collected online was exported in an anonymised format as an Excel file. We collected answers on the following questions: (1) Would you manage this child as having a serious bacterial infection? (Answers: yes/no). (2) Which diagnostics or therapeutics would you perform? (Options: no action and/or discharge; antipyretic; fluid trial; blood tests; chest-radiography; lumbar puncture; urine dipstick; oral antibiotics; intravenous antibiotics; admission). Study participants could tick as many items as they judged relevant. (3) What is 
the chance of SBI in this child? (Answer: $0-100 \%$ on a VAS $\left.\left(\operatorname{VAS}^{1}\right)\right)$ [1]. As CRP is the strongest predictor of the Feverkidstool, we studied the additional value of CRP in clinicians' management decision, with the following question: (4) A CRP is taken and returns at (continuous value) $\mathrm{mg} / \mathrm{l}$. What is the chance of SBI in this child? (Answer: 0-100\% $\operatorname{VAS}\left(\operatorname{VAS}^{2}\right)$ ).

Participant's background information was collected after finishing the video vignettes. These questions included (1) Are you a: Emergency Medicine clinician/Paediatrician; (2) How long have you been working as an Emergency Medicine clinician/paediatrician? (Options: $<5$ years; $5-10$ years; 10 15 years; $>15$ years); (3) Have you ever missed/recognised a serious infection too late? (Options: yes/no).

\section{Definitions and outcome measures}

All participants were informed about the predefined SBI definition in the letter for the study invitation: culture or radiographically proven bacterial infection (e.g. meningitis, sepsis, bacteremia, pneumonia, urinary tract infection, bacterial gastroenteritis, osteomyelitis or ethmoiditis). The outcome SBI in the vignettes was defined as management of the child as having a SBI.

Detailed descriptions on the Feverkidstool development and validation have been published earlier [19]. The originally reported discriminative ability according to the area under the receiver operating characteristic curve (AUC) of the model to predict pneumonia was 0.81 (standard error 0.04) and for other SBI 0.86 (standard error: 0.03) [19]. As the Feverkidstool was based on a polytomous logistic regression model, two risk scores were calculated, one for pneumonia and one for other SBI (e.g. urinary tract infection). We used the highest risk score in the comparison with the VAS risk scores of the video vignettes. We dichotomised the outcome of performed diagnostics and/or therapeutics. This outcome was scored 'present' if participants ticked fluid trial, blood tests, chest-radiography, lumbar puncture, urine dipstick, administration of oral/ intravenous antibiotics and/or admission. When 'no action and/or discharge and/or antipyretics' was chosen, the outcome was scored as 'not present'.

All vignettes had a statement on age, temperature and duration of fever. Abnormal clinical signs and symptoms were distributed amongst the different vignettes, with ten vignettes having one alarming sign, four vignettes with two alarming signs and seven vignettes having three or more alarming signs.

\section{Statistical analysis}

First, we assessed the range of estimated median risks by clinical judgement (VAS) and the risk with the added value of CRP. Second, we measured the patient characteristics which enact SBI management with discrete choice experiment (DCE) analysis. Finally, we compared VAS risk scores with prediction model based judgement (Feverkidstool).

DCEs are a quantitative approach to assess preferences for e.g. medical interventions and are increasingly used in health care [10]. In DCEs, it is assumed that important items influencing medical interventions, such as vital signs, can be described by its characteristics (i.e. attributes) [24]. Those characteristics are further specified by variants of that characteristics (i.e. attribute levels). A second assumption is that the levels of those attributes are determined by the individuals' preference for a medical intervention [24]. We studied the clinical variables of the Feverkidstool (www.erasmusmc.nl/feverkidstool) as attributes to the decision whether or not to manage febrile children of the vignettes as a SBI [13]. All DCE data was analysed by taking each choice amongst the two management alternatives as an observation. Using the Nlogit software http://www.limdep.com/ to the next sentence, the observations were analysed by a logit model. As there was a lack of diversity amongst the clinical variables 'oxygen saturation' and 'tachypnoea' between the vignettes, we could not analyse these variables accordingly. The variables tachycardia and prolonged capillary refill were taken together as one clinical variable as their correlation was too high. The influence of the different variable coefficients was tested for statistical significance ( $p$ value $\leq 0.05$ ). As at this moment, no formal statistical methods to determine sample sizes for DCE exist; our study strived to reach at least 40 respondents in line with previous studies $[6,26]$.

\section{Results}

Of the 104 invited participants, $50.4 \%$ agreed to participate and $42(40.4 \%)$ participants finished the online video vignettes. The 42 final participants included $83 \%$ paediatricians and $17 \%$ paediatric emergency medicine physicians. Fifty per cent of the participants had a working experience of more than 10 years. Almost half of the participants had at least once missed or delayed recognised serious infection (Table 1).

\section{Study intervention—video vignettes}

In Table 2, clinical characteristics of the video vignettes are summarised. Median age of the children was 12.0 months (interquartile range (IQR) 2.0-72.0), $57 \%$ were boys and the median C-reactive protein level (CRP) was $60 \mathrm{mg} / \mathrm{l}$ (IQR $10.0-110.0$ ). Answers on the four questions of the video vignettes are summarised in Table 3. Forty-one per cent of the video vignettes are managed as having a SBI according to the participants. Diagnostics and/or therapeutics were started in 
Table 1 Demographics

\begin{tabular}{ll}
\hline & Participants $(n=42)$ \\
\hline Specialism $^{\mathrm{a}}$ & \\
$\quad$ Paediatric emergency medicine clinician & $7(16.7)$ \\
$\quad$ Paediatrician & $35(83.3)$ \\
Years of working experience $^{\mathrm{a}}$ & \\
$\quad<5$ years & $4(9.5)$ \\
$5-10$ year & $17(40.5)$ \\
$10-15$ years & $9(21.4)$ \\
$>15$ years & $12(28.6)$ \\
Missed/recognised a serious infection too late & \\
$\quad$ Yes & $19(45.2)$ \\
No & $23(54.8)$ \\
\hline
\end{tabular}

${ }^{\mathrm{a}}$ Absolute number (percentage)

$77 \%$ of the video vignettes. Median risk before the knowledge of CRP $\left(\mathrm{VAS}^{1}\right.$ ) was $20.0 \%$ (IQR 9.0-50.0) and with

Table 2 Clinical variables

Video vignettes $(n=21)$

\begin{tabular}{|c|c|}
\hline \multicolumn{2}{|l|}{ Clinical variables } \\
\hline Age (months) ${ }^{\mathrm{a}}$ & $12.0(2.0-72.0)$ \\
\hline$\leq 3$ months & $4(19.0)$ \\
\hline$>3$ months $-<1$ year & $6(28.6)$ \\
\hline$\geq 1$ year $-\leq 18$ months & $5(23.8)$ \\
\hline$>18$ months & $6(28.6)$ \\
\hline Sex, male* & $12(57.1)$ \\
\hline Temperature $\left({ }^{\circ} \mathrm{C}\right)$ & $38.7(38.5-40.2)$ \\
\hline $38.5-38.9^{\circ} \mathrm{C}$ & $12(57.1)$ \\
\hline $39.0-39.9^{\circ} \mathrm{C}$ & $7(33.3)$ \\
\hline$\geq 40.0^{\circ} \mathrm{C}$ & $2(9.5)$ \\
\hline Duration fever ${ }^{\mathrm{a}}$ (days) & $2.0(1.0-3.0)$ \\
\hline Prolonged capillair refill ${ }^{*}(>2 \mathrm{~s})$ & $4(19.0)$ \\
\hline Chest wall retractions ${ }^{*}$ & $3(14.3)$ \\
\hline Ill appearance ${ }^{*}$ & $7(33.3)$ \\
\hline Saturation $\left(<94 \% \mathrm{O}_{2}\right)^{*}$ & $1(4.8)$ \\
\hline Respiratory rate $^{\mathrm{a}}$ (/minute) & $32.0(20.0-60.0)$ \\
\hline Tachypnoea & $1(4.8)$ \\
\hline Heart rate $^{\mathrm{a}}$ (/minute) & $132.0(100.0-172.0)$ \\
\hline Tachycardia & $4(19.0)$ \\
\hline $\mathrm{CRP}^{\mathrm{a}}(\mathrm{mg} / \mathrm{L})$ & $60.0(10.0-110.0)$ \\
\hline$<40 \mathrm{mg} / \mathrm{l}$ & $8(38.1)$ \\
\hline$\geq 40 \mathrm{mg} / \mathrm{l}$ & $7(33.3)$ \\
\hline$\geq 80 \mathrm{mg} / 1$ & $6(28.6)$ \\
\hline \multicolumn{2}{|l|}{ Presence of no. alarming symptoms ${ }^{\mathrm{a}}$} \\
\hline$\leq 1$ & $11(0-1)$ \\
\hline$>1$ & $10(2-5)$ \\
\hline
\end{tabular}

*Absolute number (percentage)

${ }^{a}$ Median (min; max)
CRP information the risk $\left(\mathrm{VAS}^{2}\right)$ increased to $30.0 \%$ (IQR 10.0-60.0). As CRP values were already available in the first video for vignette 3 and 21, no change in risk could be measured. Details of performed diagnostics, therapeutics and follow-up are described in Table 4. More diagnostics and/or therapeutics were performed when the child was managed as SBI. Antipyretics were given in $65 \%$ of the video vignettes with no differences when stratifying by outcome $\left(\mathrm{SBI}^{\mathrm{M}}\right)$. In $94 \%$ of the video vignettes who were managed as SBI, blood tests were done and $71 \%$ were hospitalised (Table 4).

\section{Clinical judgement versus different levels of CRP}

In Fig. 1, the differences in clinical risk scores are visualised versus different levels of CRP values. The median clinical risk differences $\left(\mathrm{VAS}^{2}-\mathrm{VAS}^{1}\right)$ were positively correlated with a higher level of CRP $\left(\mathrm{SBI}^{\mathrm{M}}\right.$ yes: Pearson correlation 0.53 $(p=0.000)$ and $\mathrm{SBI}^{\mathrm{M}}$ no: Pearson correlation $0.68(p=$ $0.000)$ ). Risk scores of children classified initially already as being managed as SBI were influenced only by high levels of CRP $(>65 \mathrm{mg} / \mathrm{l})$, whereas children not managed initially as SBI were influenced by lower CRP levels (>40 mg/l) (Fig. 1).

\section{Discrete choice experiment—video vignettes}

Discrete choice experiment was based upon 20 video vignettes as the clinical variables of one video were too correlated. Almost all clinical variables of the Feverkidstool could be tested with DCE analysis, except for CRP, oxygen saturation and tachypnoea. Ranking and coefficients of influencing variables on management decision of febrile children according to the DCE analysis are presented in Table 5. All tested clinical variables influenced the decision on management of febrile children significantly. Ill appearance and the combined variables of prolonged capillary refill and tachycardia were the most influencing factors and age and duration of fever the least influencing factors.

\section{Risk scores video vignettes_risk scores Feverkidstool}

The median clinical risk score (VAS ${ }^{2}$ ) according to the participants amongst those video vignettes who were assigned as managed as SBI was $60.0 \%$ (IQR 30.0-80.5) compared to a risk score according to the Feverkidstool of $12.7 \%$ (IQR 7.728.1) (Table 6). When the video vignettes were not managed as SBI, the clinical risk score $\left(\mathrm{VAS}^{2}\right)$ amounted to $16.0 \%$ (5.0-32.0) compared to a risk of $7.3 \%(5.7-16.3)$ according the Feverkidstool (Table 7). The largest risk score differences between the vignettes and risk scores according to the Feverkidstool were seen for video vignettes with (various levels of) decreased consciousness or agitation. This item is 
Table 3 Answers of 42 participants on 21 video vignettes $\left(n_{\text {total }}=882\right)$

\begin{tabular}{|c|c|c|c|c|c|c|}
\hline & Alarming symptoms & Question 1 & Question 2 & Question 3 & & Question 4 \\
\hline Video vignette & No. & $\mathrm{SBI}^{\mathrm{M}}$ & $\mathrm{Dx} / \mathrm{Tx}^{*}$ & $\operatorname{VAS}^{1 \mathrm{a}}(\%)$ & $\mathrm{CRP}^{\mathrm{a}}(\mathrm{mg} / \mathrm{l})$ & $\operatorname{VAS}^{2 \mathrm{a}}(\%)$ \\
\hline 1 & 2 & $3(7.1)$ & $16(38.1)$ & $10.0(4.8-20.0)$ & 85 & $26.5(10.0-44.8)$ \\
\hline 2 & 1 & $29(69.0)$ & $42(100.0)$ & $30.0(20.0-50.3)$ & 70 & $54.5(30.0-79.3)$ \\
\hline 3 & 1 & $11(26.2)$ & $26(61.9)$ & $16.0(7.8-32.8)$ & 38 & $10.0(4.8-23.0)$ \\
\hline 4 & 3 & $27(64.3)$ & $39(92.9)$ & $27.0(10.0-51.8)$ & 100 & $60.0(30.8-76.0)$ \\
\hline 5 & 3 & $41(97.6)$ & $42(100.0)$ & $81.0(60.0-90.0)$ & 65 & $71.5(50.0-90.0)$ \\
\hline 6 & 3 & $13(31.0)$ & $36(85.7)$ & $20.5(10.0-40.0)$ & 90 & $44.0(20.0-69.3)$ \\
\hline 7 & 1 & $23(54.8)$ & $33(78.6)$ & - & 10 & $30.5(11.0-60.3)$ \\
\hline 8 & 1 & $27(64.3)$ & $41(97.6)$ & $30.0(14.0-50.0)$ & 25 & $17.0(10.0-29.3)$ \\
\hline 9 & 1 & $4(9.5)$ & $25(59.5)$ & $10.0(4.021 .0)$ & 30 & $9.5(4.0-21.0)$ \\
\hline 10 & 2 & $41(97.6)$ & $42(100.0)$ & $80.0(62.5-90.0)$ & 50 & $69.5(40.0-90.0)$ \\
\hline 11 & 4 & $9(21.4)$ & $38(90.5)$ & $10.5(5.0-21.0)$ & 90 & $40.5(21.0-69.0)$ \\
\hline 12 & 1 & $5(11.9)$ & $32(76.2)$ & $10.5(5.8-21.0)$ & 28 & $6.0(4.0-14.5)$ \\
\hline 13 & 1 & $0(0)$ & $11(26.2)$ & $5.0(2.8-15.5)$ & 36 & $4.0(0.8-12.0)$ \\
\hline 14 & 6 & $16(38.1)$ & $38(90.5)$ & $16.0(9.8-40.0)$ & 60 & $30.0(16.3-50.0)$ \\
\hline 15 & 3 & $32(76.2)$ & $42(100.0)$ & $41.5(20.0-69.3)$ & 75 & $62.5(38.5-80.0)$ \\
\hline 16 & 1 & $1(2.4)$ & $15(35.7)$ & $8.5(2.8-15.8)$ & 10 & $1.0(0.0-6.0)$ \\
\hline 17 & 3 & $41(97.6)$ & $42(100.0)$ & 82.5 (69.8-93. 3) & 48 & $81.5(49.8-91.8)$ \\
\hline 18 & 2 & $7(16.7)$ & $32(76.2)$ & $11.5(7.8-25.3)$ & 110 & $60.0(31.0-80.0)$ \\
\hline 19 & 1 & $9(21.4)$ & $24(57.1)$ & $15.5(8.3-30.0)$ & 75 & $30.5(19.3-50.0)$ \\
\hline 20 & 1 & $16(38.1)$ & $35(83.3)$ & $21.0(10.0-45.5)$ & 35 & $13.5(8.0-36.3)$ \\
\hline 21 & 2 & $10(23.8)$ & $29(69.0)$ & - & 100 & $19.5(6.8-30.3)$ \\
\hline Total & & $365 / 882(41.4)$ & $680 / 882(77.1)$ & $20.0(9.0-50.0)$ & $60.0(35.0-85.0)$ & $30.0(10.0-61.0)$ \\
\hline
\end{tabular}

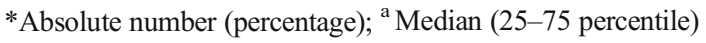

Question 1: Would you manage this child as having a serious bacterial infection?

$\mathrm{SBI}^{\mathrm{M}}$ : child is managed as having SBI according to participant

Question 2: Which diagnostics or therapy would you perform?

Dx/Tx: diagnostics and/ or therapy done (defined as: fluid trial; blood tests; chest-radiography; lumbar puncture; urine dipstick; administration of oral/ intravenous antibiotics or admission)

Question 3: What is the chance of SBI in this child? (Answer: 0-100 \% on a VAS (VAS ${ }^{1}$ ))

VAS $^{1}$ : risk assessment without knowledge of CRP (0-100 \% VAS)

Question 4: A CRP is taken and returns at (continuous value) mg/l. What is the chance of SBI in this child? (Answer: 0-100 \% VAS (VAS ${ }^{2}$ )

$\mathrm{VAS}^{2}$ : risk assessment with knowledge of CRP (0-100 \% VAS)

clearly observed when watching the video vignettes, but this clinical variable is not included in the predictors of the Feverkidstool. Finally, no differences were found in median clinical risk scores when stratified for previously missed diagnoses of the participant ( $p=0.218$ ).

\section{Discussion}

\section{Main findings}

This is the first study on real life video vignettes to determine febrile child characteristics which enact clinicians' management decisions. High clinical risk scores to manage febrile children as SBI were created by clinicians. All tested clinical variables of the Feverkidstool influenced clinicians' management decisions of febrile children significantly with ill appearance and aberrant circulatory signs being the most important. Moderate CRP levels influenced risk scores in children who were initially not managed as SBI whereas high CRP levels were needed to influence risk scores in children who were initially already managed as SBI. In children managed as SBI risk thresholds judged by the clinician were higher compared with predicted risk thresholds according to the Feverkidstool. Clinical risk thresholds of children not managed as having a SBI were more comparable to prediction model-based risk thresholds. 
Table 4 Diagnostics, therapy and follow-up

\begin{tabular}{llll}
\hline Diagnostics & $\mathrm{SBI}^{\mathrm{M}}$ yes $n=365$ & $\mathrm{SBI}^{\mathrm{M}}$ no $n=517$ & $N_{\text {total }}=882$ \\
No diagnostics & $4(1.1)$ & $100(19.3)$ & $104(11.8)$ \\
Urine dipstick & $252(69.0)$ & $134(25.9)$ & $386(43.8)$ \\
Fluid trial & $135(37.0)$ & $73(14.1)$ & $208(23.6)$ \\
Blood tests & $344(94.2)$ & $180(34.8)$ & $524(59.4)$ \\
Chest-radiography & $112(30.7)$ & $76(14.7)$ & $188(21.3)$ \\
Lumbar puncture & $140(38.4)$ & $9(1.7)$ & $149(16.9)$ \\
Therapy and follow-up & $\mathrm{SBI}^{\mathrm{M}}$ yes $n=365$ & $\mathrm{SBI}^{\mathrm{M}}$ no $n=517$ & $574(65.1)$ \\
Antipyretics & $244(66.8)$ & $330(63.8)$ & $478(54.2)$ \\
No therapy & $74(20.3)$ & $404(78.1)$ & $27(3.1)$ \\
Oral antibiotics & $11(3.0)$ & $16(3.1)$ & $213(24.1)$ \\
Intravenous antibiotics & $209(57.3)$ & $4(0.8)$ & $354(40.1)$ \\
Admission & $258(70.7)$ & $96(18.6)$ & $480(54.4)$ \\
Discharge & $75(20.5)$ & $405(78.3)$ & \\
\hline
\end{tabular}

\section{Comparison with literature}

In this study, we aimed to get insight in patient characteristics and contextual factors influencing management decisions of the febrile child at the ED. One way to approach this process of diagnostic reasoning is decision making [11]. Decision making has been influenced by statistical models of reasoning under uncertainty using pre- and post-test probability according to Bayes' theorem. This model deals with two major classes of errors in clinical reasoning: in the assessment of either pretest probability or the strength of the evidence [11]. Although the pretest probability of having SBI (prevalence of disease) is depending on several factors as for example age and relevant medical history, the pretest probability determined by health care setting was considered stable in the vignettes. However, we focused on the interpretation of clinicians' strengths of evidence of the probability of a serious infection. For this decision process, we performed discrete choice experiment (DCE) analysis, which is an increasingly used method applied
Fig. 1 Relation video vignettes risk difference and $\mathrm{C}$-reactive protein $(\mathrm{mg} / \mathrm{l})$

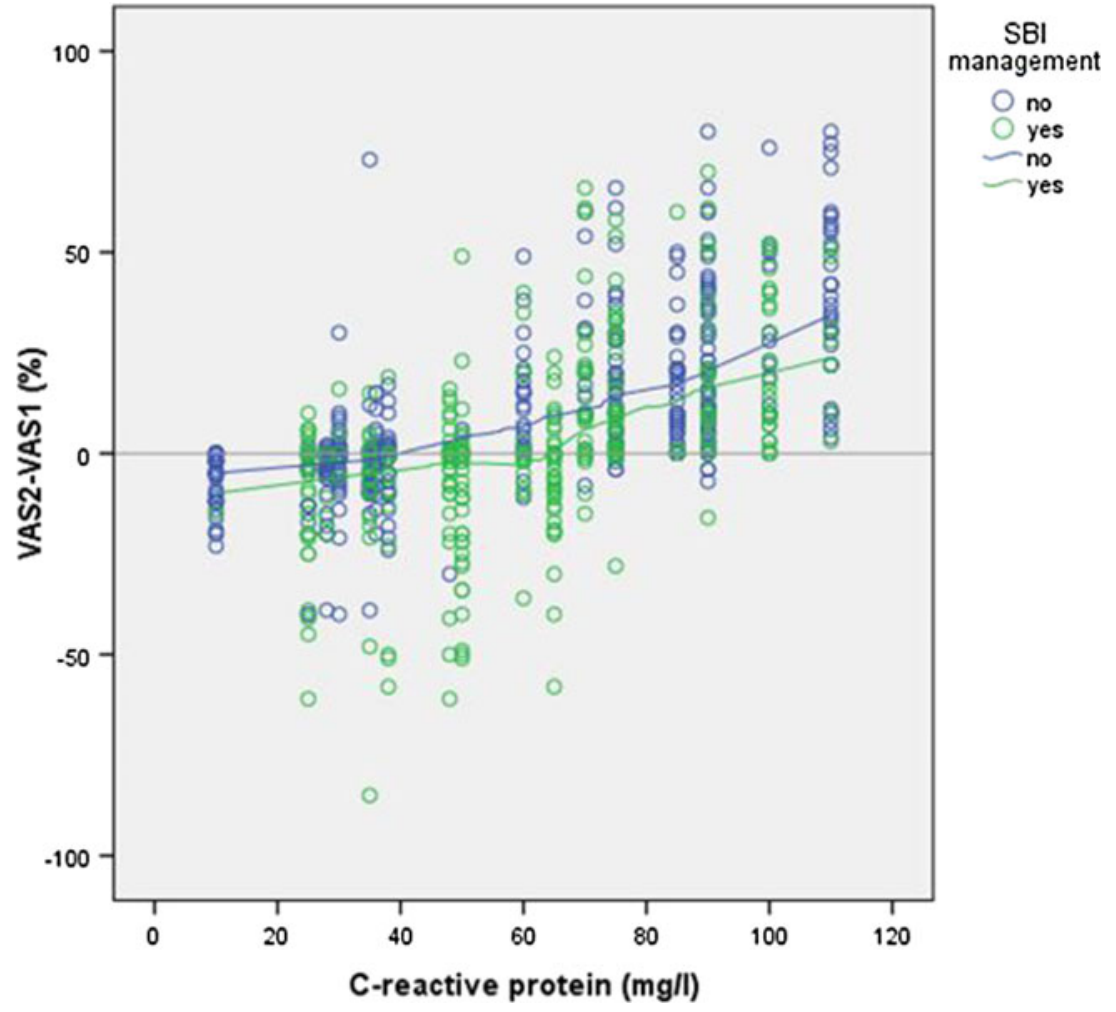


Table 5 Influencing variables on management decisions in febrile children $\left(\mathrm{SBI}^{\mathrm{M}}\right)$ : a discrete choice experiment $\left(n_{\text {total }}=882\right)$

\begin{tabular}{llll}
\hline Clinical variables & Ranking & Coefficients (SE) & $p$ value \\
\hline Intercept & & $-0.92(0.37)$ & 0.013 \\
Ill appearance & 1 & $1.15(0.13)$ & $<0.001$ \\
Prolonged capillary refill $(>2$ s) and/or tachycardia & 2 & $0.99(0.17)$ & $<0.001$ \\
Chest wall retractions & 3 & $-0.97(0.22)$ & $<0.001$ \\
Temperature $\left(\geq 39.0{ }^{\circ} \mathrm{C}\right)$ & 4 & $0.77(0.12)$ & $<0.001$ \\
Sex $($ male $)$ & 5 & $0.63(0.11)$ & $<0.001$ \\
Duration fever $($ days $)$ & 6 & $0.51(0.20)$ & 0.009 \\
Age $(\geq 1$ year) & 7 & $-0.42(0.12)$ & 0.001 \\
Saturation $\left(<94 \% \mathrm{O}_{2}\right)$ & NA & NA & NA \\
Tachypnoea & NA & NA & NA \\
\hline
\end{tabular}

$\mathrm{SBI}^{\mathrm{M}}$ : child is managed as having $\mathrm{SBI}$ according to participant

$N A$ not applicable, items could not been tested with DCE analyses in studies where clinicians weigh clinical information in the diagnostic work-up [3].

In literature on diagnostic reasoning, evidence-based medicine is the most successful educational method in the

Table 6 Clinical risk scores (video vignettes) versus prediction model risk scores (Feverkidstool) in children managed as $\mathrm{SBI}\left(\mathrm{SBI}^{\mathrm{M}}=\right.$ yes $)$

\begin{tabular}{|c|c|c|}
\hline Video vignettes (no.) & $\begin{array}{l}\operatorname{VAS}^{2}(\%)^{\mathrm{a}} \\
\mathrm{SBI}^{\mathrm{M}} \text { yes } n=365\end{array}$ & $\begin{array}{l}\text { Feverkidstool }(\%)^{\mathrm{a}} \\
n=365\end{array}$ \\
\hline & Risk $\leq 10 \%$ & \\
\hline 12 & $5.0(2.0-9.5)$ & 16.3 \\
\hline \multirow[t]{2}{*}{13} & - & - \\
\hline & Risk $10-50 \%$ & \\
\hline 16 & $15.0(15.0-15.0)$ & 2.0 \\
\hline 8 & $20.0(12.0-30.0)$ & 8.9 \\
\hline 3 & $23.0(9.0-61.0)$ & 7.2 \\
\hline 20 & $29.0(12.5-61.8)$ & 3.8 \\
\hline 9 & $30.5(8.3-66.3)$ & 11.6 \\
\hline \multirow[t]{2}{*}{14} & $47.0(32.0-76.8)$ & 36.9 \\
\hline & Risk $\geq 50 \%$ & \\
\hline 21 & $54.0(17.8-80.3)$ & 12.7 \\
\hline 19 & $59.0(45.0-90.0)$ & 7.3 \\
\hline 2 & $60.0(30.0-80.0)$ & 38.2 \\
\hline 7 & $60.0(30.0-72.0)$ & 2.3 \\
\hline 1 & $62.0(50.0-62.0)$ & 20.6 \\
\hline 6 & $68.0(35.0-83.0)$ & 19.0 \\
\hline 15 & $68.0(52.3-80.8)$ & 50.5 \\
\hline 10 & $70.0(44.5-90.0)$ & 7.7 \\
\hline 11 & $70.0(57.5-81.0)$ & 4.8 \\
\hline 4 & $71.0(35.0-80.0)$ & 9.7 \\
\hline 5 & $72.0(50.0-90.0)$ & 22.2 \\
\hline 18 & $80.0(21.0-82.0)$ & 6.6 \\
\hline 17 & $83.0(50.0-92.5)$ & 28.1 \\
\hline Total & $60.0(30.0-80.5)$ & $12.7(7.7-28.1)$ \\
\hline
\end{tabular}

translation of statistical decision theory into clinical practice [25]. Within this translation, we aimed to elaborate on the determination of quantitative decision thresholds that proved to be a complex topic. Most studies used optimised performance measures as area under the receiver operating characteristic curve (AUC) or sensitivity/specificity to establish these thresholds. Other studies described Delphi procedures to determine their clinical based cutoff points $[5,18,20,22$, 32]. In our study, we described clinicians' assigned median risk estimates according to which patients would have been managed as SBI. We observed agreement on clinical and prediction model-based risk thresholds when clinicians decided not to manage the febrile child as a SBI. However, the clinical risk threshold to manage the child as SBI was much higher compared with prediction model-based judgement. This phenomenon is well recognised, as clinicians don't want to miss serious, but treatable diseases, there is a tendency to overestimate the probability of these diseases [11].

\section{Clinical and research implications}

The most important finding of this study includes the high risk scores clinicians assigned to those children who they would have managed as SBI (median risk $60.0 \%$ (IQR 30-80.5)). This observation is in contrast to our hypothesis that very low risk thresholds might be chosen for specific diagnosis with high morbidity/mortality (e.g. meningitis). Apparently, clinicians create more dichotomous risk estimations (high risk or low risk) for the management of specific serious infections with reassessment of risk estimates after every diagnostic step. Clinicians used a stepwise approach in the management of febrile children, rather than considering one risk thresholds for SBI in general. We observed agreement in predictive value of all tested clinical predictor variables in the detection of children with SBI, for both clinical-based as prediction model-based judgement. Clinicians were guided by ill 
Table 7 Clinical risk scores (video vignettes) versus prediction model risk scores (Feverkidstool) children not managed as $\mathrm{SBI}\left(\mathrm{SBI}^{\mathrm{M}}=\right.$ no)

\begin{tabular}{|c|c|c|}
\hline $\begin{array}{l}\text { VAS }^{1}(\%)^{\mathrm{a}} \text { Feverkidstool } \\
(\%)^{\mathrm{a}} \text { Video vignettes (no.) }\end{array}$ & $\mathrm{SBI}^{\mathrm{M}}$ no $n=517$ & $n=517$ \\
\hline & Risk $\leq 10 \%$ & \\
\hline 16 & $1.0(0.0-5.5)$ & 2.0 \\
\hline 13 & $4.0(0.8-12.0)$ & 5.7 \\
\hline 12 & $6.0(4.0-16.5)$ & 16.3 \\
\hline 9 & $8.5(4.0-16.3)$ & 11.6 \\
\hline 3 & $10.0(3.0-17.0)$ & 7.2 \\
\hline 8 & $10.0(6.0-18.0)$ & 8.9 \\
\hline \multirow[t]{2}{*}{20} & $10.0(7.0-20.3)$ & 3.8 \\
\hline & Risk $10-50 \%$ & \\
\hline 10 & $13.0(13.0-13.0)$ & 7.7 \\
\hline 21 & $15.5(5.3-28.0)$ & 12.7 \\
\hline 7 & $17.0(10.0-28.0)$ & 2.3 \\
\hline 1 & $20.0(10.0-39.0)$ & 20.6 \\
\hline 14 & $20.0(10.0-31.3)$ & 36.9 \\
\hline 17 & $20.0(20.0-20.0)$ & 28.1 \\
\hline 19 & $25.0(15.5-48.0)$ & 7.3 \\
\hline 11 & $30.0(20.0-53.5)$ & 4.8 \\
\hline 5 & $40.0(40.0-40.0)$ & 22.2 \\
\hline 6 & $40.0(17.5-57.5)$ & 19.0 \\
\hline 2 & $42.0(33.0-74.5)$ & 38.2 \\
\hline 4 & $46.0(22.0-60.0)$ & 9.7 \\
\hline \multirow[t]{2}{*}{15} & $46.5(26.0-64.5)$ & 50.5 \\
\hline & Risk $\geq 50 \%$ & \\
\hline 18 & $60.0(31.0-71.0)$ & 6.6 \\
\hline Total & $16.0(5.0-32.0)$ & $7.3(5.7-16.3)$ \\
\hline
\end{tabular}

appearance and aberrant circulatory signs in their febrile child evaluation, which were not the most influencing factors according to the Feverkidstool. For the Feverkidstool respiratory predictors as chestwall retractions and oxygen saturation were more powerful influencing factors. Furthermore, we found that CRP levels influenced clinical risk scores differently in children with or without initial SBI management, with higher influence of clinical factors than of CRP value. In our study population, this approach was not enhanced by experiences of errors in the past. These insights in influencing factors in the clinical prediction of febrile children at risk for SBI helps us to understand, review and evaluate clinical management decisions.

Compared to prediction model based risk scores, thresholds of children who were not managed as having a SBI were more comparable, ranging from 7 to $16 \%$. We might have to conclude that this risk threshold is justified as SBI rule-out threshold, but no agreement can be defined on rule-in thresholds as there appears too much difference between prediction model and the clinical stepwise risk assessment in children managed as SBI.

\section{Strengths and limitations}

The main strength of this study is the use of real-life videos instead of paper-case patients. This approach is a more representative way of portraying real life, and there is an evolving evidence base on the use of patient video cases as educational interventions [8, 23].

A second strength of the study is the use of the Feverkidstool as an arithmetic model to compare the subjective overall assessment of the clinician when evaluating the febrile child. In a review describing vignette studies on medical decision behaviour, it was concluded that most studies on this topic did not compare their results to some sort of normative benchmark [3]. Moreover, the role of prediction models becomes greater, as clinicians may increasingly rely on alarming signs and symptoms described in (inter)national clinical guidelines and prediction models due to decreasing incidence of SBI. Although, there was a discrepancy in risk assessment of some video vignettes (e.g. vignettes 7,11 and 18), probably due to the absence of variables as decreased consciousness or agitation in the Feverkidstool.

There are some other limitations in this study. Videos still lack some aspects of real life such as observation time or concise descriptions of patients' history. However, from literature, we know that more detailed case descriptions will be assigned a higher subjective probability of disease than a brief abstract of the same case, even if they contain the same disease information [11]. Another limitation includes the determination of some clinical variables by the clinicians' judgement (ill appearance, chestwall retractions and capillary refill time). In this way, misclassification of these clinical predictors could have occurred. However, this approach does reflect clinical practice and therefor may just strengthen generalisability of our results.

Next, the DCE analysis had to be performed within the availability of a limited number of video vignettes. As a consequence, we were forced to exclude or merge some predictor variables (e.g. oxygen saturation and tachypnoea) to meet the DCE theory design. Second, although a response rate of $50 \%$ for clinicians was similar to other DCE studies, this response rate is not optimal [2, 9, 29]. However, due to the experienced background of all participants, we assume limited answer variability resulting in representative study results.

\section{Conclusion}

In this study on real-life video vignettes, we observed high risk scores in clinicians' risk estimation of SBI management in febrile children, and these risks are mostly influenced by the clinical characteristics ill appearance and aberrant circulatory signs. Uniform risk thresholds at which one should start SBI management in febrile children remains unclear, as the 
concept of clinicians' dichotomous risk thresholds was hardly comparable to the overall SBI risk assessment of the prediction model. However, more consistent results were found for clinical and prediction model-based risk thresholds at which we refrain from SBI management in the febrile child visiting the emergency department.

Acknowledgments We gratefully acknowledge all participants of the video vignettes study for their time, patience and complete participation in our study. We want to thank Paul Muston for the collaboration and the development of the video vignettes for practical use. We acknowledge Johan van der Lei, Ewout Steyerberg and Yvonne Vergouwe for discussion on the approach towards DCE analyses.

Authors'contributions EdVK, DR, ML and HAM substantially contributed to the conception and design of the study. DR collected the original video vignettes as used in the study. He monitored participant response rates and undertook data extraction. EdVK actively enrolled study participants and monitored response rates. She undertook data extraction and performed data analysis. She drafted the initial manuscript. EdBG was responsible for the DCE analysis and interpretation. RO and HAM participated and supervised analysis and interpretation of the data. All authors reviewed and revised the manuscript and approved the final manuscript as submitted.

\section{Compliance with ethical standards}

Conflict of interest The authors declare that they have no competing interests.

Funding source EK is supported by ZonMW, a Dutch organisation for health research and development. The study sponsor had no role in study design, in the collection, analysis, and interpretation of data; in the writing of the report; nor in the decision to submit the paper for publication.

Financial disclosure Dr. Damian Roland is the co-director of QuackApps, a mobile applications company which designed the online risk assessment system. No payments were made for the delivery of the video vignettes. The other authors have no financial disclosures relevant to this article.

Open Access This article is distributed under the terms of the Creative Commons Attribution 4.0 International License (http:// creativecommons.org/licenses/by/4.0/), which permits unrestricted use, distribution, and reproduction in any medium, provided you give appropriate credit to the original author(s) and the source, provide a link to the Creative Commons license, and indicate if changes were made.

\section{References}

1. Aitken RC (1969) Measurement of feelings using visual analogue scales. Proc R Soc Med 62:989-993

2. Ashcroft DM, Seston E, Griffiths CE (2006) Trade-offs between the benefits and risks of drug treatment for psoriasis: a discrete choice experiment with U.K. dermatologists. Br J Dermatol 155:12361241

3. Bachmann LM, Muhleisen A, Bock A, ter Riet G, Held U, Kessels AG (2008) Vignette studies of medical choice and judgement to study caregivers' medical decision behaviour: systematic review. BMC Med Res Methodol 8:50

4. Bachur RG, Harper MB (2001) Predictive model for serious bacterial infections among infants younger than 3 months of age. Pediatrics 108:311-316

5. Bell LM, Grundmeier R, Localio R, Zorc J, Fiks AG, Zhang X, Stephens TB, Swietlik M, Guevara JP (2010) Electronic health record-based decision support to improve asthma care: a clusterrandomized trial. Pediatrics 125:e770-777

6. Berchi C, Dupuis JM, Launoy G (2006) The reasons of general practitioners for promoting colorectal cancer mass screening in France. Eur J Health Econ 7:91-98

7. Craig JC, Williams GJ, Jones M, Codarini M, Macaskill P, Hayen A, Irwig L, Fitzgerald DA, Isaacs D, McCaskill M (2010) The accuracy of clinical symptoms and signs for the diagnosis of serious bacterial infection in young febrile children: prospective cohort study of 15781 febrile illnesses. BMJ 340:c1594

8. D. R, T. B (2015) Using patient video cases in medical education. Arch Disd Edu Pract Accepted for publication

9. de Bekker-Grob EW, Bliemer MC, Donkers B, Essink-Bot ML, Korfage IJ, Roobol MJ, Bangma CH, Steyerberg EW (2013) Patients' and urologists' preferences for prostate cancer treatment: a discrete choice experiment. Br J Cancer 109:633-640. doi:10. 1038/bjc. 2013.370

10. de Bekker-Grob EW, Ryan M, Gerard K (2012) Discrete choice experiments in health economics: a review of the literature. Health Econ 21:145-172

11. Elstein AS, Schwartz A (2002) Clinical problem solving and diagnostic decision making: selective review of the cognitive literature. BMJ 324:729-732

12. Galetto-Lacour A, Zamora SA, Andreola B, Bressan S, Lacroix L, Da Dalt L, Gervaix A (2010) Validation of a laboratory risk index score for the identification of severe bacterial infection in children with fever without source. Arch Dis Child 95:968-973

13. Hensher D, Rose J, Green W (2005) Applied choice analysis: a primer. Cambridge University Press, Cambridge: UK

14. Kuppermann N, Fleisher GR, Jaffe DM (1998) Predictors of occult pneumococcal bacteremia in young febrile children. Ann Emerg Med 31:679-687

15. McGinn TG, Guyatt GH, Wyer PC, Naylor CD, Stiell IG, Richardson WS, Evidence-Based Medicine Working Group (2000) Users' guides to the medical literature: XXII: how to use articles about clinical decision rules. Jama 284:79-84

16. Najaf-Zadeh A, Dubos F, Pruvost I, Bons-Letouzey C, Amalberti R, Martinot A (2011) Epidemiology and aetiology of paediatric malpractice claims in France. Arch Dis Child 96:127-130

17. Nelson DS, Walsh K, Fleisher GR (1992) Spectrum and frequency of pediatric illness presenting to a general community hospital emergency department. Pediatrics 90:5-10

18. Nigrovic LE, Kuppermann N, Macias CG, Cannavino CR, MoroSutherland DM, Schremmer RD, Schwab SH, Agrawal D, Mansour KM, Bennett JE, Katsogridakis YL, Mohseni MM, Bulloch B, Steele DW, Kaplan RL, Herman MI, Bandyopadhyay S, Dayan P, Truong UT, Wang VJ, Bonsu BK, Chapman JL, Kanegaye JT, Malley R, Pediatric Emergency Medicine Collaborative Research Committee of the American Academy of Pediatrics (2007) Clinical prediction rule for identifying children with cerebrospinal fluid pleocytosis at very low risk of bacterial meningitis. JAMA 297: $52-60$

19. Nijman R, Vergouwe Y, Thompson M, Veen van M, Meurs Van A, Lei van der J, Steyerberg E, Moll H, Oostenbrink R (2013) Clinical prediction model to aid emergency doctors managing febrile children at risk of serious bacterial infections: diagnostic study. BMJ 346:f1706

20. Oostenbrink R, Oostenbrink JB, Moons KG, Derksen-Lubsen G, Essink-Bot ML, Grobbee DE, Redekop WK, Moll HA (2002) 
Cost-utility analysis of patient care in children with meningeal signs. Int J Technol Assess Health Care 18:485-496

21. Pantell RH, Newman TB, Bernzweig J, Bergman DA, Takayama JI, Segal M, Finch SA, Wasserman RC (2004) Management and outcomes of care of fever in early infancy. JAMA 291:1203-1212

22. Reilly BM, Evans AT, Schaider JJ, Das K, Calvin JE, Moran LA, Roberts RR, Martinez E (2002) Impact of a clinical decision rule on hospital triage of patients with suspected acute cardiac ischemia in the emergency department. Jama 288:342-350

23. Roland D, Coats T, Matheson D (2012) Towards a conceptual framework demonstrating the effectiveness of audiovisual patient descriptions (patient video cases): a review of the current literature. BMC Med Educ 12:125

24. Ryan M (2004) Discrete choice experiments in health care. BMJ 328:360-361

25. Sackett DL (1997) Evidence-based medicine and treatment choices. Lancet 349:570, author reply 572-573

26. Salkeld G, Solomon M, Butow P, Short L (2005) Discrete-choice experiment to measure patient preferences for the surgical management of colorectal cancer. Br J Surg 92:742-747

27. Sim I, Gorman P, Greenes RA, Haynes RB, Kaplan B, Lehmann H, Tang PC (2001) Clinical decision support systems for the practice of evidence-based medicine. J Am Med Inform Assoc 8:527-534
28. Slater M, Krug SE (1999) Evaluation of the infant with fever without source: an evidence based approach. Emerg Med Clin North Am 17:97-126, viii-ix

29. Szeinbach SL, Harpe SE, Williams PB, Elhefni H (2008) Testing for allergic disease: parameters considered and test value. BMC Fam Pract 9:47

30. Thompson M, Coad N, Harnden A, Mayon-White R, Perera R, Mant D (2009) How well do vital signs identify children with serious infections in paediatric emergency care? Arch Dis Child 94: 888-893

31. Van den Bruel A, Aertgeerts B, Bruyninckx R, Aerts M, Buntinx F (2007) Signs and symptoms for diagnosis of serious infections in children: a prospective study in primary care. Br J Gen Pract 57: 538-546

32. Wang CJ, McGlynn EA, Brook RH, Leonard CH, Piecuch RE, Hsueh SI, Schuster MA (2006) Quality-of-care indicators for the neurodevelopmental follow-up of very low birth weight children: results of an expert panel process. Pediatrics 117:2080-2092

33. Wolfe I, Thompson M, Gill P, Tamburlini G, Blair M, van den Bruel A, Ehrich J, Pettoello-Mantovani M, Janson S, Karanikolos M, McKee M (2013) Health services for children in western Europe. Lancet 381:1224-1234

34. Yen K, Gorelick MH (2007) Strategies to improve flow in the pediatric emergency department. Pediatr Emerg Care 23:745-749, quiz 750-741 